\title{
Pleuropericardial mesotheliomas: A report of two unusual
}

\section{cases}

\begin{abstract}
Mesothelioma is an aggressive malignant tumor of the mesothelium. The majority of mesotheliomas arise from the pleura. However, less commonly mesothelioma can arise in other locations such as peritoneal, pericardium, or tunica vaginalis testis. We report two rare cases here, one with pericardial mesothelioma and another with malignant pleural mesothelioma with synchronous colonic carcinoma. The clinical, radiological, and pathology findings in these cases are discussed along with a brief review of pertinent literature.
\end{abstract}

Keywords: Carcinoma colon association, computed tomography, mesothelioma, pericardial, pleural, synchronous carcinoma

\section{INTRODUCTION}

Mesothelioma, in general, is an aggressive malignant tumor of the mesothelium. The majority of mesotheliomas arise from the pleura. However, less commonly, mesothelioma can arise in other locations such as peritoneal, pericardium, or tunica vaginalis testis owing to the presence of the mesothelial tissue in different parts of the body.

Mesotheliomas usually develop in individuals over 60 years of age, but rarely, it has also been reported to occur in children. A large majority of these cases are associated with prior asbestos exposure. The incidence of malignant pleural mesothelioma in people without prior exposure to asbestos is around 1 in a million population, whereas in people with a history of prior asbestos exposure it is as high as 10 in 100 persons. $^{[1]}$

Malignant pericardial mesothelioma accounts for approximately $2 \%-3 \%$ of all primary pericardial tumors. ${ }^{[2]}$ It is the most common primary malignant neoplasm of the pericardium and does not have any association with asbestos exposure.

We report here two cases of malignant mesotheliomas one of primary pericardial origin and the other of pleural origin

\begin{tabular}{|l|c|}
\hline \multicolumn{2}{|c|}{ Access this article online } \\
\hline \multirow{2}{*}{$\begin{array}{l}\text { Website: } \\
\text { www.asjo.in }\end{array}$} & Quick Response Code \\
\hline \multirow{2}{*}{ DOI: } & 回 \\
10.4103/ASJO.ASJO_41_17 & \\
& \\
\hline
\end{tabular}

with no history of any asbestos exposure in the past. In addition, the case with malignant pleural mesothelioma had a coexisting colonic malignancy, an extremely rare chance occurrence.

\section{CASE REPORTS}

\section{Case 1}

A 45-year-old female presented with progressive shortness of breath, abdominal distension, and swelling of feet over 2 months. On examination, bilateral pleural and pericardial effusions were detected. Her electrocardiogram showed low voltage complexes and an echocardiography (ECHO) performed subsequently confirmed a large pericardial effusion with signs of cardiac tamponade. Pericardiocentesis was performed immediately, and $1 \mathrm{~L}$ of hemorrhagic fluid was drained.

\section{harsha Vardhana Kuruba Ramanjaneyulu, Anu Kapoor, Chinmayee Biswal, Phani Chakravrty Mutnuru \\ Department of Radiology and Imageology, Hyderabad, Telangana, India \\ Address for correspondence: Dr. Harsha Vardhana Kuruba Ramanjaneyulu, \\ Nizam's Institute of Medical Sciences, \\ Hyderabad, Telangana, India. \\ E-mail: drharsha_kr@yahoo.co.in}

This is an open access journal, and articles are distributed under the terms of the Creative Commons Attribution-NonCommercial-ShareAlike 4.0 License, which allows others to remix, tweak, and build upon the work non-commercially, as long as appropriate credit is given and the new creations are licensed under the identical terms.

For reprints contact: reprints@medknow.com

How to cite this article: Ramanjaneyulu HK, Kapoor A, Biswal C, Mutnuru PC. Pleuropericardial mesotheliomas: A report of two unusual cases. Asian J Oncol 2018;4:25-9. 
Pericardial fluid analysis suggested exudative nature of fluid but was negative for acid-fast bacilli. Chest and abdominal sonography showed bilateral mild-to-moderate pleural effusions with moderate ascites. The patient was further investigated with contrast-enhanced computed tomography (CT) of the chest and abdomen [Figure 1] which demonstrated moderate pericardial effusion along with a peripherally enhancing nodular soft-tissue density lesion measuring $4.4 \mathrm{~cm} \times 3.2 \mathrm{~cm}$ in size, along the pericardium around the right atrium. In addition, a few subcentimeter sized lymph nodes were noted in paracardiac location. Bilateral moderate pleural effusions were also present. However, no pleural or lung nodules were found. There were significant ascites in the abdomen. In view of these imaging findings, a possible diagnosis of malignant pleuropericardial effusion was considered.

Following radiological suspicion, USG-guided biopsy of the pericardial mass lesion was performed and sent for histopathology analysis. Histopathological examination of the biopsied specimen [Figure 2] showed neoplastic cells arranged in cords, trabeculae as well as glandular structures. The neoplastic cells were predominantly epithelioid cells arranged in clusters. The cells were polygonal with scanty to moderate amount of eosinophilic cytoplasm and round to oval dark nuclei. There was mild anisonucleosis. Immunohistochemistry showed positive mesothelial markers (calretinin, CK 5/6 and cytokeratin) and negative pulmonary epithelial markers (TTF-1). Thus, a diagnosis of pericardial mesothelioma, (epithelioid variety) was confirmed. The patient was thereafter referred to the department of oncology for further management.

\section{Case 2}

A 42-year-old male presented with fever, shortness of breath, and left-sided chest pain for 3 months. He also had altered bowel habits, abdominal pain, headache, and double
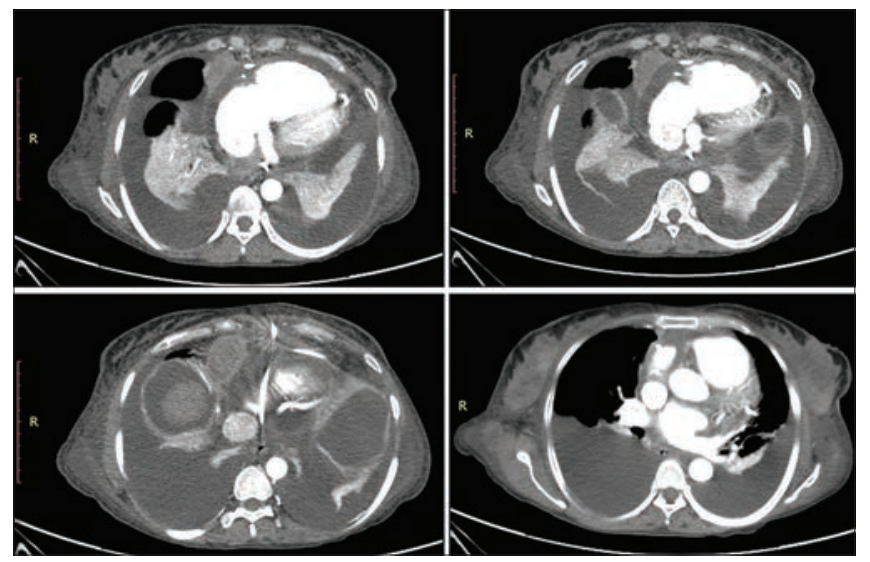

Figure 1: Contrast-enhanced computed tomography chest images showing a well-defined enhancing mass lesion along the right anterolateral aspect of pericardium with moderate pericardial effusion and bilateral pleural effusions with underlying collapse of the lungs vision for 3 months. There was associated history of weight loss. He was evaluated outside where his chest radiograph showed a homogeneous lobulated extrapulmonary opacity in the left hemithorax causing volume loss of the left lung. A thoracocentesis was performed from the left side and the pleural fluid aspirated showed increased cell count with lymphocyte predominance. He was started on anti-tubercular therapy which he took for 3 months without any relief of symptoms. Due to worsening of symptoms, he was referred to a higher center where positron emission tomography CT (PET-CT) [Figure 3] was performed. PET-CT revealed left-sided pleural thickening with nodularity and heterogeneous enhancement along the mediastinal, costal, and diaphragmatic surface with pockets of loculated effusion at places. The nodular thickened pleura was hypermetabolic with avid fluorodeoxyglucose uptake. In addition, there were multiple mediastinal lymph nodes showing uptake of the isotope. In addition, another synchronous metabolically active polypoidal lesion was detected in the distal descending colon on PET-CT. It was thought that the metabolically active lesion in the colon was a primary malignant neoplasm and the pleural nodular lesion was metastatic from the colonic malignancy. Hence, a colonoscopy and biopsy were performed along with CT-guided biopsy of pleural lesion.

Surprisingly, the histopathology from pleural biopsy showed infiltrating and superficial collections of cells that formed papillary structures with identifiable fibrovascular cores. There were cells forming tubules and many single, infiltrating cells. The tumor cells had hyperchromatic, round to polygonal nuclei with irregular nuclear rims and prominent nucleoli. Immunohistochemistry showed positive mesothelial markers (calretinin, CK5/6, and cytokeratin) and negative

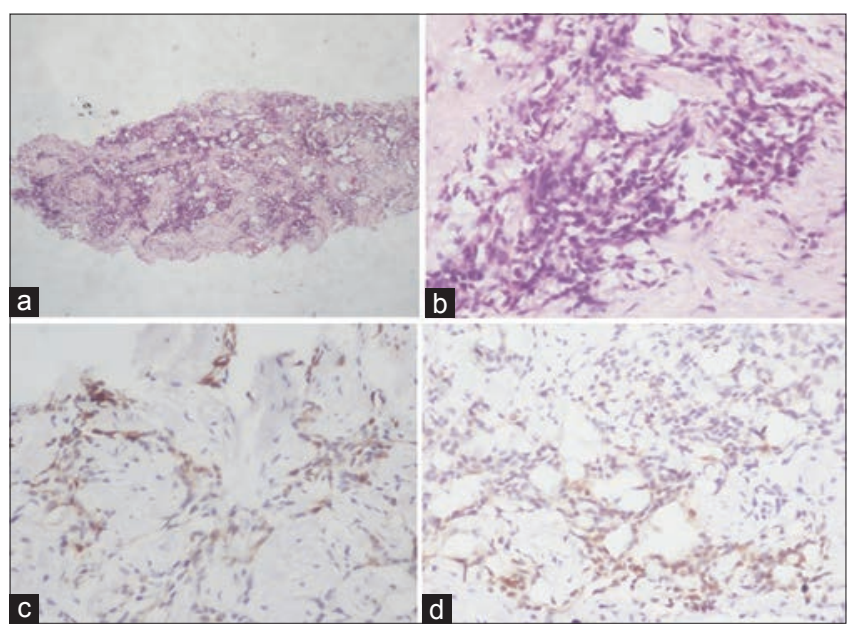

Figure 2: ( $a$ and b) Histopathology of mesothelioma. (a) Neoplastic cells arranged in cords, trabeculae, glandular structures $(\times 40)$. (b) The cells show epithelioid morphology with moderate amount of eosinophilic cytoplasm and round to oval dark nuclei ( $\times 400)$. (c and d) Immunohistochemistry. (c) Calretinin positivity $(\times 400),(d)$ cytokeratin $5 / 6$ positivity in neoplastic cells $(\times 400)$ 

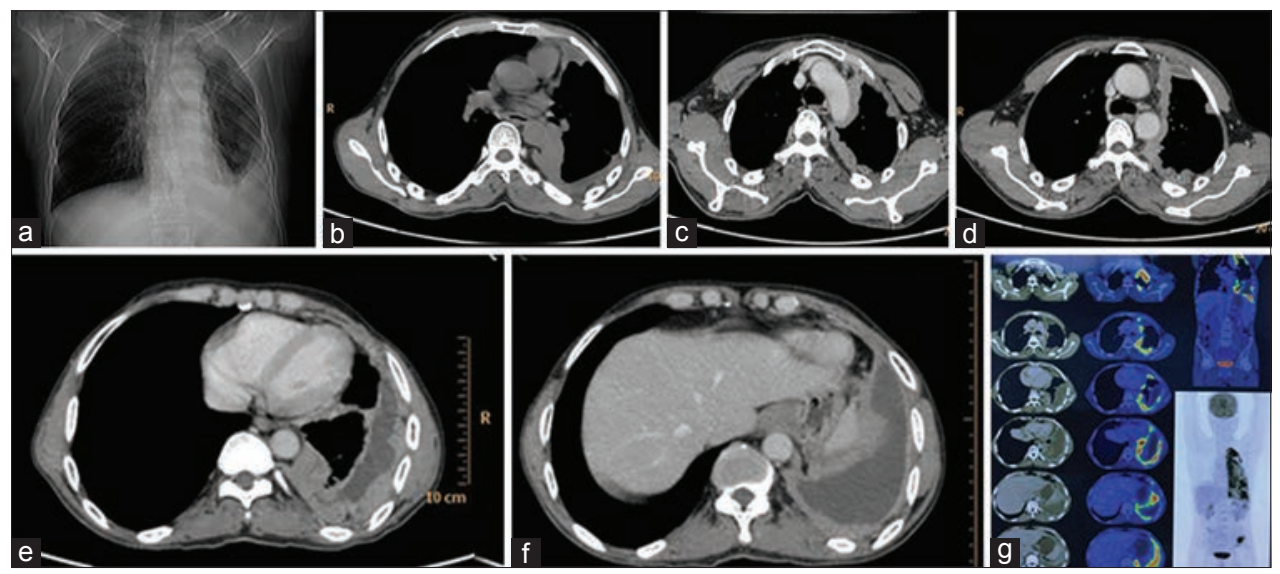

Figure 3: Scanogram (a) showing opacity in the periphery of left lower lung zone silhouetting left costophrenic angle, left dome diaphragm, nonenhanced computed tomography axial image (b) showing peripheral nodular pleural thickening which is showing heterogeneous enhancement in contrast-enhanced computed tomography images (c-f) with pleural effusion. Positron Emission tomography images (g) showing metabolically active heterogeneously enhancing thickening with nodularity involving the mediastinal, costal, and diaphragmatic left pleura with loculated areas of effusion at places

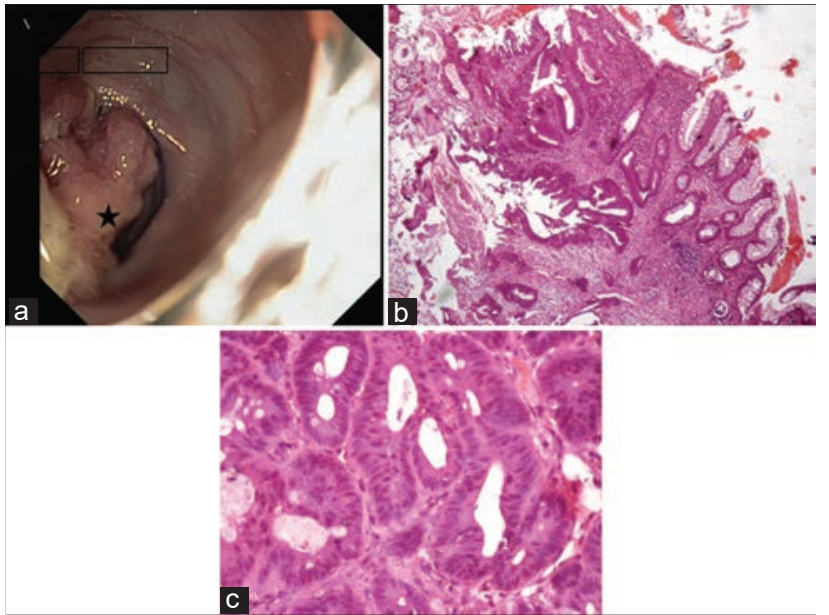

Figure 4: Colonoscopy image. (a) Showing a nodular, friable polypoidal growth (Black star) in descending colon. (b) Normal colonic mucosa in transition to a lesion in the lamina propria infiltrating the muscularis mucosae ( $\mathrm{H}$ and $\mathrm{E}, \times 40)$. (c) Closely packed glands lined by pseudostratified layers of cuboidal to columnar cells showing nuclear pleomorphism and hyperchromasia ( $H$ and $E, \times 400)$

pulmonary epithelial markers (TTF-1). Hence, a diagnosis of pleural mesothelioma was made.

Sections from colonic mucosal biopsy [Figure 4] showed a lesion comprising of glandular structures. These were seen infiltrating the smooth muscle bundles and showed stratification and atypia of the lining epithelium. Immunohistochemistry was positive for CDX2, patchy positivity for CK20, and negative for CK7 thereby confirming the diagnosis of colonic adenocarcinoma.

\section{DISCUSSION}

Mesothelioma is an aggressive malignant tumor of the mesothelium. About $65 \%-70 \%$ of malignant mesotheliomas arise from pleura and 30\% from peritoneum, whereas mesothelioma originating from the pericardium or tunica vaginalis testis is rare and accounts for $1 \%-2 \%$ of all mesotheliomas. ${ }^{[3]}$

Unlike pleural mesotheliomas, which have a proven association with prior asbestos exposure. ${ }^{[4]}$ The association of pericardial mesothelioma with asbestos is not well established.

The patients with pleural mesothelioma typically present with dyspnea and nonpleuritic chest pain. Pleural effusions are seen in a large majority of patients at some stage during their disease. The clinical presentation of pericardial mesothelioma is mainly that of acute pericarditis, constrictive pericarditis or cardiac tamponade $\mathrm{e}^{[5]}$ and may sometimes mimic coronary artery disease.

While pericardial mesothelioma presents as a localized mass associated with pericardial effusion ${ }^{[6]}$ or as a lobulated thickening of the pericardium, the appearance of pleural mesothelioma is that of a soft-tissue attenuation nodular mass which spreads along pleural surfaces including into pleural fissures and often creating a pleural rind. It has a predilection for a direct invasion of adjacent structures such as chest wall, diaphragm, and mediastinum. ${ }^{[7]}$

Distant metastasis from pericardial mesothelioma is very rare. However, pleural mesotheliomas frequently metastasize to the contralateral lung and locoregional lymph nodes. ${ }^{[7]}$

The diagnosis of mesothelioma may require support from immunohistochemistry and positive calretinin and CK5/6 test are suggestive of mesothelioma. ${ }^{[8]}$ 
Surgical resection remains the main treatment option for primary pericardial mesothelioma. The prognosis of this disease remains extremely poor with a median survival time of about 6 months due to its late presentation, the inability of complete tumor eradication by surgery and the poor response of primary pericardial mesothelioma to radiotherapy or chemotherapy. Recently, newer chemotherapeutic regimens following complete excision of the tumor have shown prolonged survival times. ${ }^{\mid 9]}$

The treatment of pleural mesothelioma includes extrapleural pneumonectomy, adjuvant chemotherapy, and radiotherapy. The prognosis is poor for all tumor types with a median overall survival without treatment of 4-12 months. ${ }^{[10]}$ Even with aggressive multimodality therapy overall 5-year survival remains poor $(3 \%-18 \%)$ with a median survival time of approximately 18 months. ${ }^{[10]}$

In our second case, a concurrent colonic adenocarcinoma was detected along with malignant pleural mesothelioma. This is an extremely rare occurrence. The risk factors associated with the development of mesothelioma are asbestos, prior radiation ports, exposure to irritants (thorium, talc, erionite, or mica), familial Mediterranean fever ${ }^{[11]}$ and the common postulated associations with the development of colonic adenocarcinoma include transformation from adenomatous polyps, familial adenomatous polyposis, dietary factors, smoking, family history of colon cancer, and inflammatory bowel disease. To the best of our knowledge, no common associations have been found in the etiopathogenesis of development of colonic adenocarcinoma and malignant pleural mesothelioma.

Occasional occurrence of peritoneal mesothelioma and colonic adenocarcinoma has been reported previously in literature. ${ }^{[12]}$ The coexistence of colonic adenocarcinoma and pleural mesothelioma in our case fulfills the diagnostic criteria of multiple primary malignant neoplasia, which is a rare entity. ${ }^{[13]}$ The two tumors showed distinctive morphology and were seen to arise from different tissues of origin as evidenced by histology and immunohistochemical studies. The diagnosis of such tumors is made possible with the combined use of radiopathologic techniques such as CT, PET, histopathology, and immunohistochemistry. ${ }^{[14]}$

Following imaging workup of the second case, if only a colonic biopsy had been performed, the pleural lesions would have been misinterpreted as metastatic from the colonic primary and over staged the disease. Performing biopsies from both colonic as well as pleural lesions confirmed two separate primary malignancies occurring synchronously. The point to be learned from this case is to keep in mind that pleural mesothelioma can coexist with a second neoplasm elsewhere in the body and accurate tissue diagnosis is crucial for the staging of disease.

\section{CONCLUSION}

Imaging plays an important role in the evaluation of malignant pleural and pericardial mesotheliomas. CT is widely used as the initial imaging modality for diagnosis and staging of mesothelioma of pleura and pericardium. Magnetic resonance imaging and more recently, PET has proved helpful in further delineating the extent of disease and targeting biopsy sites, especially in cases where surgical management is contemplated. The new IHC markers help to differentiate mesothelioma from lung neoplasms and metastatic disease. Each imaging modality has its advantages and limitations, but careful clinical, radiological, and pathological correlation is useful in determining the most appropriate treatment options for these patients.

A review of the existing literature shows that the presence of synchronous colonic malignancy along with primary pleural mesothelioma is an exceedingly rare occurrence, but never the less must be considered as a differential diagnosis when the two lesions are detected simultaneously. Tissue examination from both lesions with immunohistochemistry is essential to identify different morphologic patterns and confirm the different organs of tumor origin as it is critical for a definitive diagnosis.

\section{Declaration of patient consent}

The authors certify that they have obtained all appropriate patient consent forms. In the form the patient(s) has/have given his/her/their consent for his/her/their images and other clinical information to be reported in the journal. The patients understand that their names and initials will not be published and due efforts will be made to conceal their identity, but anonymity cannot be guaranteed.

\section{Financial support and sponsorship}

Nil.

\section{Conflicts of interest}

There are no conflicts of interest.

\section{REFERENCES}

1. Antman KH. Natural history and epidemiology of malignant mesothelioma. Chest 1993;103:373S-6S.

2. Karadžić R, Kostić-Banović L, Antović A, Celar M, Katić V, Iliæ G, et al. Primary pericardial mesothelioma presenting as constrictive pericarditis. Arch Oncol 2005;13:150-2. 
3. Falkenstern-Ge RF, Kimmich M, Bode-Erdmann S, Friedel G, Ott G, Kohlhäufl M, et al. Pleural mesothelioma presenting as periumbilical metastasis: The first clinical documentation. Case Rep Oncol Med 2013;2013:198729.

4. Naidich DP, Srichai MB, Krinsky GA. Computed Tomography and Magnetic Resonance of the Thorax. Philadelphia, PA, USA: Lippincott Williams and Wilkins; 2007.

5. Kirwan M, Blake S, Neligan M, O’Malley E, Bergin A, Bonar F, et al. Cardiac constriction due to malignant disease of the pericardium. Ir J Med Sci 1983;152:454-5.

6. Gössinger HD, Siostrzonek P, Zangeneh M, Neuhold A, Herold C, Schmoliner R, et al. Magnetic resonance imaging findings in a patient with pericardial mesothelioma. Am Heart J 1988;115:1321-2.

7. Wang ZJ, Reddy GP, Gotway MB, Higgins CB, Jablons DM, Ramaswamy M, et al. Malignant pleural mesothelioma: Evaluation with CT, MR imaging, and PET. Radiographics 2004;24:105-19.

8. Hyun TS, Barnes M, Tabatabai ZL. The diagnostic utility of D2-40, calretinin, CK5/6, desmin and MOC-31 in the differentiation of mesothelioma from adenocarcinoma in pleural effusion cytology. Acta Cytol 2012;56:527-32.
9. Taylor P, Castagneto B, Dark G, Marangolo M, Scagliotti GV, van Klaveren RJ, et al. Single-agent pemetrexed for chemonaïve and pretreated patients with malignant pleural mesothelioma: Results of an international expanded access program. J Thorac Oncol 2008;3:764-71.

10. Zielinski M, Hauer J, Hauer L, Pankowski J, Nabialek T, Szlubowski A, et al. Staging algorithm for diffuse malignant pleural mesothelioma. Interact Cardiovasc Thorac Surg 2010;10:185-9.

11. Pelin K, Hirvonen A, Linnainmaa K. Expression of cell adhesion molecules and connexins in gap junctional intercellular communication deficient human mesothelioma tumour cell lines and communication competent primary mesothelial cells. Carcinogenesis 1994;15:2673-5.

12. Xie W, Green LK, Patel RA, Lai S., A Case of Unsuspected Peritoneal Mesothelioma Occurring with Colonic Adenocarcinoma Masquerading as Peritoneal Metastases, Case Rep Pathol v.2014;2014:865. doi: $10.1155 / 2014 / 838506$.

13. Warren $\mathrm{S}$, Gates O. Multiple primary malignant tumors: A survey of the literature and statistical study. Am J Cancer 1932;16:1358-403.

14. Eisenstaedt JS. Multiple primary malignant tumors. J Am Med Assoc 1938;110:2056-9. 